\title{
Postextrasystolic potentiation in the isolated rat heart ${ }^{1}$
}

F. L. MEIJLER, F. v. D. BOGAARD,L. H. v. D. TWEEL, AND D. DURRER

University Department of Cardiology and Clinical Physiology, Wilhelmina-

Gasthuis, Amsterdam; and Laboratory of Medical Physics, University of Amsterdam, The Netherlands

Meijler, F. L., F. v.D. Bogaard, L. H. v.D. Tweel, And D. Durrer. Postextrasystolic potentiation in the isolated rat heart. Am. J. Physiol. 202(4): 63I-635. I 962.- "Postextrasystolic potentiation" of isotonic contractions of the intact isolated rat heart was studied. It was found that the Frank-Starling mechanism does not participate in the increase of the contraction following a premature beat and a compensatory pause. A linear relationship could be demonstrated between the prematurity of the extra beat and the amplitude of the postcompensatory contraction. The increase of amplitude of the postcompensatory contraction was found to counterbalance the decrease of the premature beat. At a constant net rate, whether the rhythm was regular or not, the average contraction height was constant. Postextrasystolic potentiation pattern following an interpolating premature beat was different from postextrasystolic potentiation following a premature beat and a compensatory pause. Postextrasystolic potentiation following an interpolating premature beat could not be distinguished from poststimulation potentiation. It was therefore concluded that postextrasystolic potentiation does not exist as a specific phenomenon.

$T_{1}$ HE IMPORTANCE OF THE RELATION of rhythm and rate to myocardial contractility is gaining increasing attention in cardiovascular studies. In all these studies isometric tension in isolated myocardial strips or pressure in isovolumic contracting ventricles are measured. Isometric tension or pressure in the isovolumic ventricle need bear no relation to ventricular ejection.

Present studies were undertaken to investigate the relationship between cycle length and the degree of myocardial shortening in isolated intact mammalian hearts.

Before going into details, it is necessary to define a number of terms derived from literature as we do not

Received for publication 3r August 196r.

1 This study was supported in part by grants from The Netherlands Organization for Pure Research (Z.W.O.), The Hague, The Netherlands. want to increase the already existing confusion in terminology.

a) Postextrasystolic potentiation (I); increase in amplitude of myocardial contraction occurring after a premature beat.

b) Poststimulation potentiation (2); increase in amplitude of myocardial contraction following a number of contractions of a higher frequency.

c) Rest contraction(s) (2); contraction(s) elicited following a period of cardiac standstill.

d) Restitution (3) of contractility; augmentation of amplitude of myocardial contraction on lengthening, and diminution of amplitude on shortening of preceding cycle length.

e) Treppe or staircase (4); increase of amplitude of myocardial contraction from nihil to a certain constant amplitude at a constant frequency, occurring after a long period of cardiac standstill.

Langendorff $(5,6)$ was the first who observed an increase of height of the contraction following a premature beat in isolated frog and mammalian hearts. This increase in contractility has been generally explained by the Frank-Starling mechanism $(7-10)$. Siebens and coworkers (I I) and Lendrum et al. (I2) recently demonstrated, however, that in the isometrically contracting canine heart, Starling's law of the heart does not explain this phenomenon.

It appears that postextrasystolic potentiation is insufficiently described. In a recent review, Hajdu and Leonard (13) gave a description of Langendorff's observation (5) of a premature beat followed by a compensatory pause and one enlarged contraction. However, in their diagram (13; p. I85, Fig. I $F$ ) representing this experiment the premature beat arises between two normal beats (interpolating premature beat) and is followed by several enlarged contractions instead of one. In Siebens' paper, potentiation of the first postextrasystolic contraction occurring after a compensatory pause is described, whereas Hoffman's study (I) deals 
with postextrasystolic potentiation following interpolating premature beat(s).

In this part of the work an attempt is made to analyze the postextrasystolic potentiation mechanism.

\section{METHODS}

Isolated hearts from $250-\mathrm{g}$ white rats were perfused at $37 \mathrm{C}$, using the Langendorff technique described previously ( $14-16)$. Changes in length of the longitudinal axis of the left ventricle were measured by displacement of a metal rod (Fig. I) (diam. $2 \mathrm{~mm}$, length $60 \mathrm{~mm}$, wt. I.7 g), connected to the apex of the heart and placed within a coil (Fig. I). The load exerted on the heart by this rod is negligible, since the heart is able to contract for hours if the weight of the rod is increased to I I.7 g. The coil is part of a bridge (Fig. I) which consists of two self-inductances (L) and two resistances $\left(\mathrm{R}_{1}\right.$ and $\left.\mathrm{R}_{2}\right)$. The movements of the rod change the impedance of the coil, producing across the output terminals of the bridge a varying a-c voltage which, after amplification and detection in a phasesensitive detector, can be recorded with an electrocardiograph. The record can be calibrated by means of a second rod connected to a miicrometer and placed within the second coil (Fig. I). Unipolar electrocardiogram was recorded simultaneously. Complete control of heart rate was achieved through stimulating electrodes placed on the right atrium for fast rates or on the area trabecularis of the right ventricle for slow rates. The stimuli were square waves of $0.5 \mathrm{ma}$, and $0.5^{-1.0-\mathrm{msec}}$ duration. The rate could be changed abruptly within any diastolic interval (circuit A, Fig. I). Premature contractions could be elicited by a variable delay circuit B in Fig. I. $(L)$.
RESULTS

A number of experiments were performed to investigate the role of the Frank-Starling mechanism in postextrasystolic increase of isotonic contraction following a compensatory pause.

In the experiment represented in Fig. 2, increase of filling of ventricular cavities during the (longer) postextrasystolic compensatory pause has been eliminated by: I) turning a stopcock, interrupting coronary per-

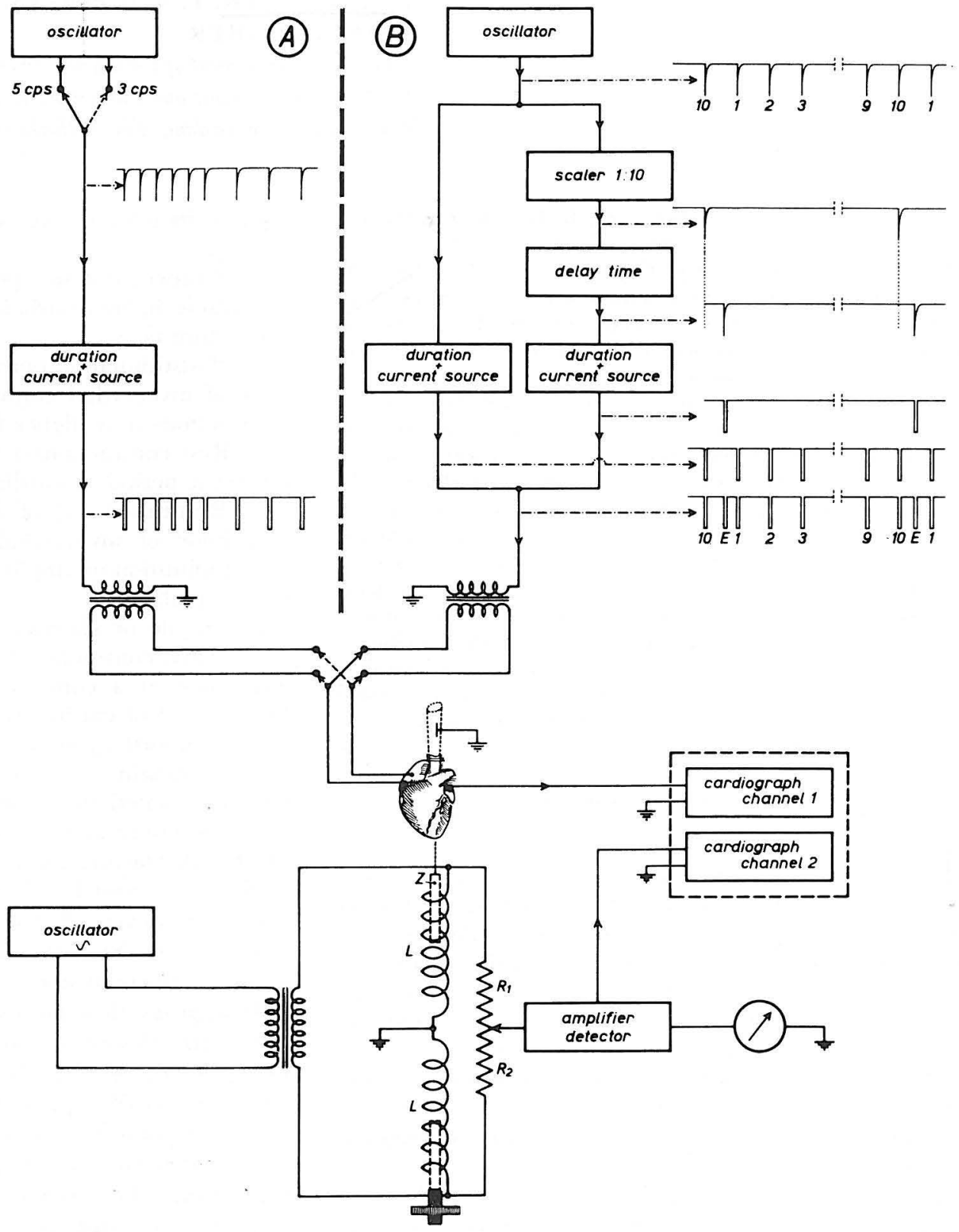

FIG. I. Diagrammatic representation of recording and stimulating equipment. Circuit $A$ serves to change stimulation rate within one diastolic interval. Circuit $B$ is used to elicit premature beats. $Z$ is a metal rod connected to the apex of the heart and positioned in the center of a coil $(L)$. The same rod, connected to a micrometer, is placed within the second coil 
fusion completely, and 2) cutting open both left and right ventricular walls, which also prevented eventual increase of transverse diameter of the heart.

Figure 2 shows left intraventricular pressure record (upper line), apical displacement d-c recording (middle), and electrocardiogram. Changes in pressure have disappeared due to the opening of the left ventricular cavity, but postextrasystolic increase of contraction,

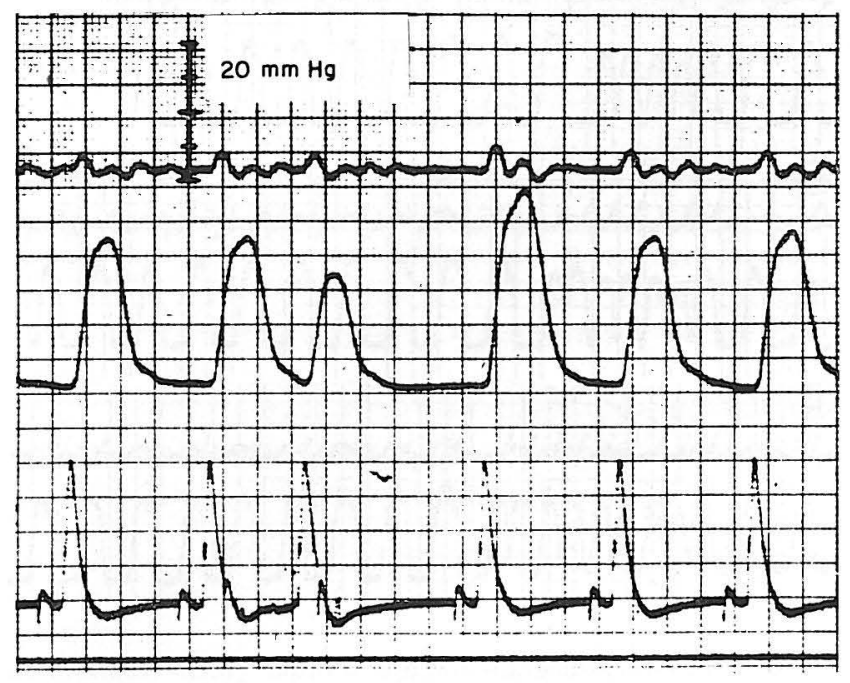

FIG. 2. Left intraventricular pressure record (upper line) and apical displacement record of an isolated rat's heart with opened ventricular cavities and interrupted coronary perfusion. Note that pressure has disappeared but that the displacement record still shows an increased height of the postextrasystolic contraction at a constant end-diastolic length. Driving frequency; $2.5 \mathrm{cycles} / \mathrm{sec}$. Delay extra stimulus; $280 \mathrm{msec}$. Paper speed; $50 \mathrm{~mm} / \mathrm{sec}$.

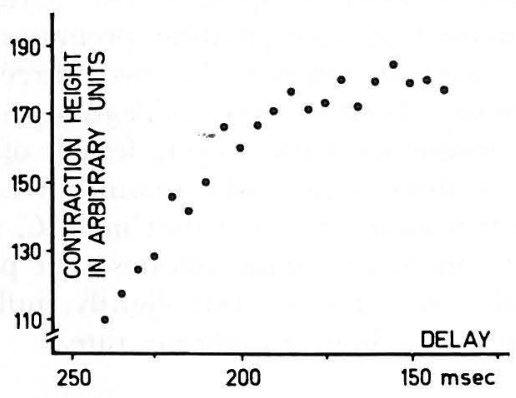

FIG. 3. Effect of delay of the extra impulse on contraction height of the first postextrasystolic beat occurring after a compensatory pause. Driving frequency; 4 cycles/sec. starting from a constant end-diastolic position, is still present.

The influence of the cycle length, preceding the extra beat, on the first subsequent postcompensatory contraction was studied by varying the delay of the extra impulse. The shorter this cycle length the longer the compensatory pause following it. Results of a representative experiment are shown in Fig. 3. It demonstrates that at a basal rate of 4 cycles/sec shortening the delay of the extra impulse (thus also lengthening the duration of the compensatory pause) increases the height of the first postextrasystolic contraction, reaching a limit at a delay of approximately $175 \mathrm{msec}$. This is due to the fact that with further shortening of the delay the extra impulse falls in the functional refractory period ( 17 ) and retards ventricular activation till the end of this period. An almost linear relationship appears to exist between the duration of the compensatory pause and the height of the subsequent contraction.

During these experiments it was also noticed that the height of the extrasystolic beat varies with its prematurity. The shorter the cycle length preceding it, the smaller the premature beat, the longer the compensatory pause, and the larger the postextrasystolic contraction.

In Table I the results are summarized of an experiment in which height of contraction of premature beat and postcompensatory contraction were measured. This table also demonstrates that the sum of the heights of the extrasystolic and the postextrasystolic contractions amounts to approximately two times the height of a preceding normal contraction. This observation prompted us to design experiments which would demonstrate that changes in cycle length, with net rate kept constant, do not change the average contraction height. Figure 4 demonstrates the results of an experiment in which, at a constant net rate of I 50 contractions/min, the regular rhythm (upper row) is gradually changed into a bigeminy with varying coupling $\left(325^{-200} \mathrm{msec}\right)$ of the extrasystolic beat. In each row the sum of the heights of all 26 contractions is approximately $480 \mathrm{~mm}$.

The experiments mentioned above deal exclusively with postextrasystolic potentiation following a compensatory pause.

Postextrasystolic potentiation pattern following an interpolating premature beat is different from postextrasystolic potentiation following a compensatory pause. This is demonstrated in Fig. 5. At a basal rate of $2.5 \mathrm{cycles} / \mathrm{sec}$ and a constant delay of the extra

TABLE I. Comparison of the sum of contraction heights of extrasystolic beat and postextrasystolic beat with two times the contraction height of a preceding normal beat

Frequency, 4 cycles/sec

\begin{tabular}{|c|c|c|c|c|c|c|c|c|c|c|c|c|c|c|c|c|c|c|}
\hline Delay, msec & 240 & 235 & 230 & 225 & 220 & 215 & 210 & 205 & 200 & $19 j$ & I9o & I 85 & 180 & I 75 & 170 & I65 & 160 & I 55 \\
\hline $\mathrm{NB}, \mathrm{mm}$ & I 5 & 16 & I 6 & I 6 & I6 & 16 & I 5.5 & 15 & 15 & I6 & I6 & 16 & I6 & I 5.5 & 15.5 & I 6 & I6 & I $5 \cdot 5$ \\
\hline $\mathrm{ES}, \mathrm{mm}$ & I 4 & 15 & I 4 & I4 & I 3.5 & I3 & 13 & 12 & 12 & I3 & I 2 & 12 & 12 & II. 5 & 12 & $\cdot 12$ & 12 & II \\
\hline PES, mm & 16 & 17 & 17 & I7 & 17 & I8 & I 8 & I 8 & I 8 & 18 & I9 & 19 & I9 & I9 & 19 & I9 & 19 & 19.5 \\
\hline$\times \mathrm{NB}, \mathrm{mm}$ & 30 & $3^{2}$ & $3^{2}$ & $3^{2}$ & $3^{2}$ & $3^{2}$ & $3^{1}$ & 30 & $3^{\circ}$ & $3^{2}$ & $3^{2}$ & $3^{2}$ & $3^{2}$ & $3^{\mathrm{I}}$ & $3^{I}$ & $3^{2}$ & $3^{2}$ & $3^{I}$ \\
\hline $\mathrm{ES}+\mathrm{PES}, \mathrm{mm}$ & 30 & $3^{2}$ & $3 \mathrm{I}$ & $3 \mathrm{I}$ & 30.5 & $3 \mathrm{I}$ & 31 & 30 & 30 & 3I & 31 & $3 I$ & $3 I$ & 30.5 & $3 \mathrm{I}$ & $3 \mathrm{I}$ & $3 \mathrm{I}$ & 30.5 \\
\hline
\end{tabular}

$\mathrm{NB}=$ normal beat ES = extrasystolic beat PES = postextrasystolic beat. 


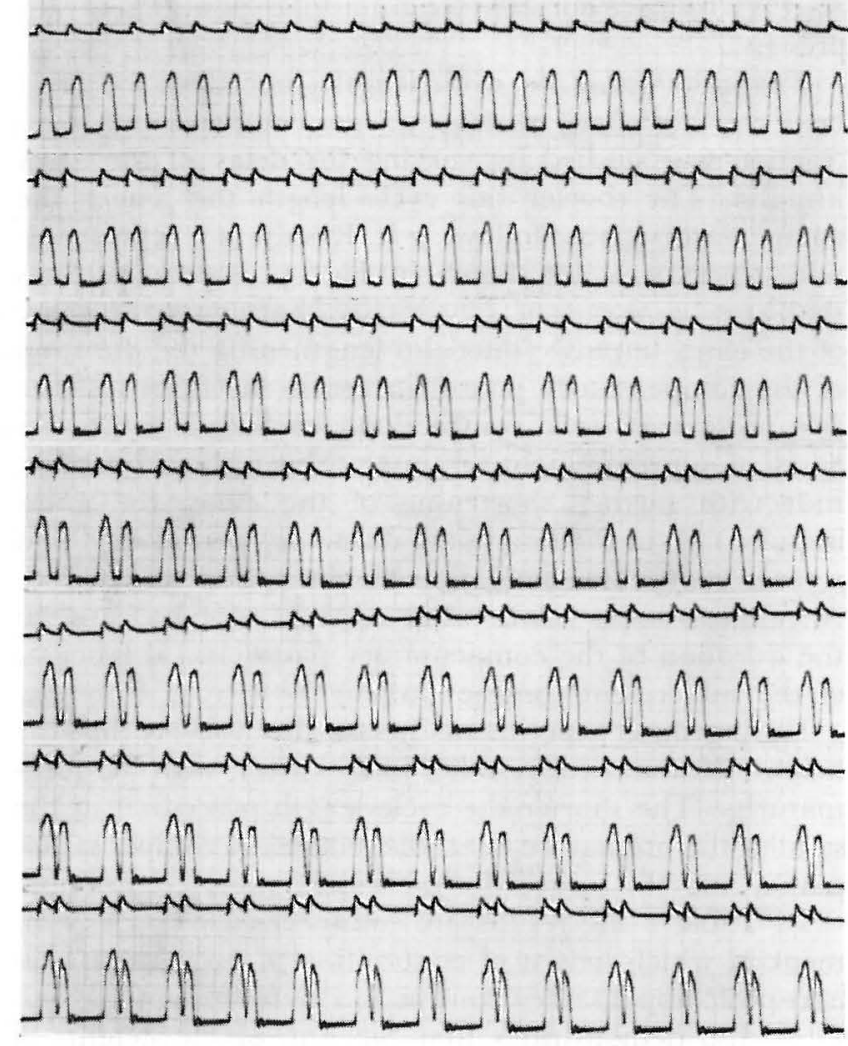

FIG. 4. Electrocardiogram and apical displacement record of an isolated perfused rat's heart. At a constant net rate the regular rhythm (upper row) is changed into a bigeminy with varying coupling (325-200 msec). Driving frequency in upper row; 2.5 cycles/sec. Paper speed; $25 \mathrm{~mm} / \mathrm{sec}$.

impulse of $240 \mathrm{msec}$, the first premature beat (double arrow) interpolates and the second one (single arrow) is followed by a compensatory pause. The interpolating premature beat is followed by one smaller contraction and a number of enlarged contractions. The premature beat followed by a compensatory pause gives rise to enlargment of the first postextrasystolic contraction only.

There is a striking resemblance between potentiation following an interpolating premature beat and post-

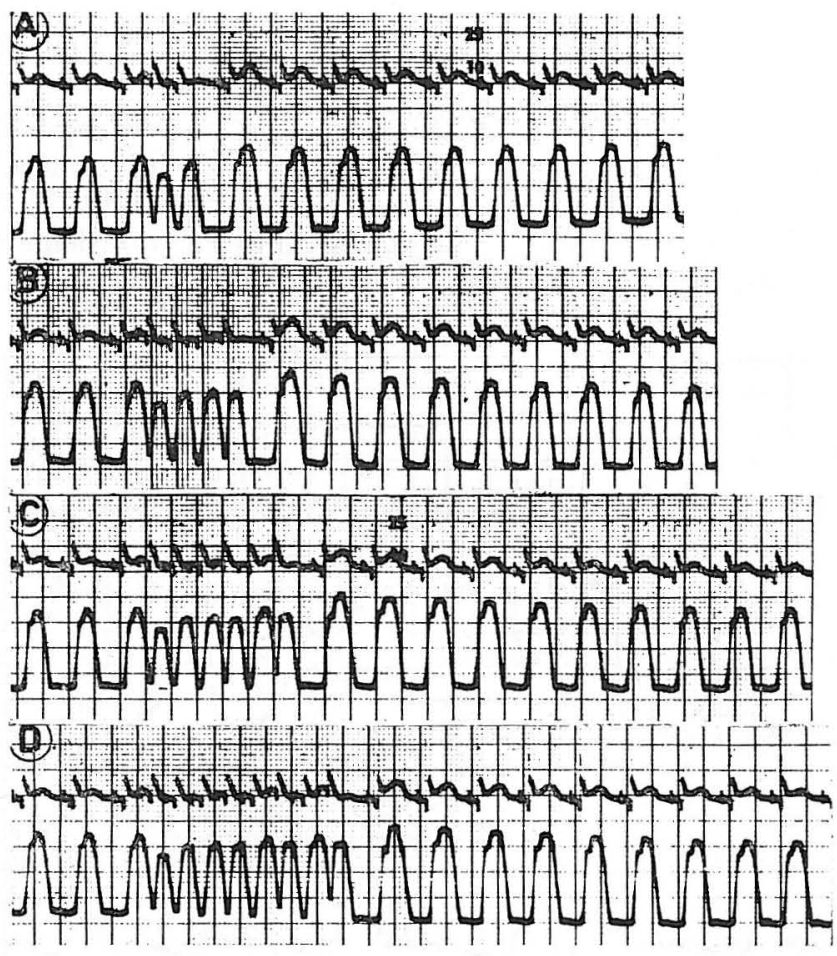

FIG. 6. Electrocardiogram and apical displacement record of an isolated perfused rat's heart. Driving frequency; 2.5 cycles/sec. Paper speed; $25 \mathrm{~mm} / \mathrm{sec}$. In $A, B, C$, and $D$, respectively, I, 2, 3 , and 4 contractions have been interpolated. Delay of the extra impulse; $200 \mathrm{msec}$. Note the similarity in potentiation in all 4 rows.

stimulation potentiation. This is illustrated in Fig. 6. In this experiment the heart was stimulated with a basal rate of 2.5 cycles/sec (cycle length $400 \mathrm{msec}$ ). In Fig. $6 \mathrm{~A}$ one extra stimulus is applied with a delay of 200 msec, originating an interpolating premature beat. In Fig. $6 B, C$, and $D$, respectively, two, three, and four contractions have been interpolated, causing a temporary increase in frequency with a cycle length of $200 \mathrm{msec}$. At the most there is a slight quantitative difference between potentiation in $A$ and that in $B, C$, and $D$.

This experiment also demonstrates that poststimulation potentiation is not, or but slightly, influenced by the duration of the high stimulation rate.

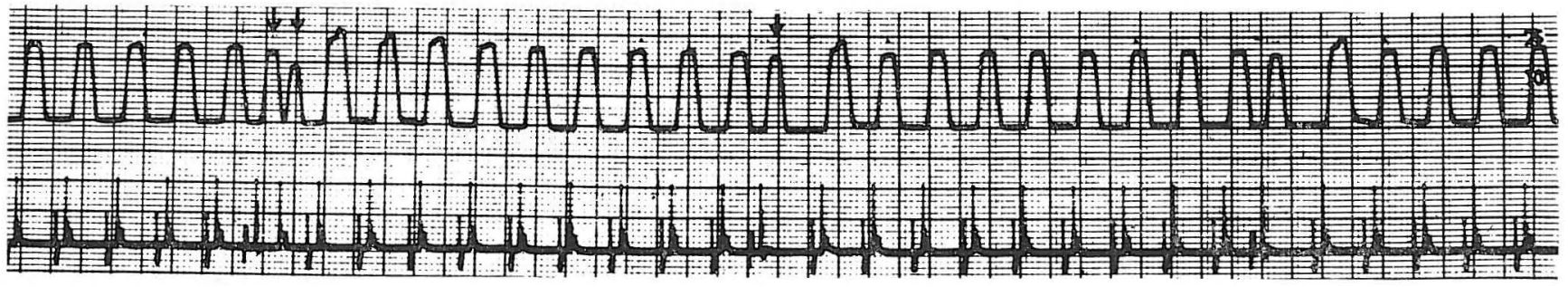

FIG. 5. Apical displacement record and electrocardiogram of an isolated perfused heart with, respectively, an interpolated premature beat (double arrow) and a premature beat followed by a compensatory pause (single arrow). The interpolated premature beat is followed by several enlarged postextrasystolic contractions with decay of potentiation. Premature beat followed by a compensatory pause elicits one enlarged postextrasystolic contraction. Paper speed; $25 \mathrm{~mm} / \mathrm{sec}$. Driving frequency; 2.5 cycles $/ \mathrm{sec}$. Delay of the extra stimulus; 240 msec. 
According to present day terminology potentiation in upper row $A$ is called postextrasystolic potentiation and potentiation in the lower rows is called poststimulation potentiation.

\section{DISCUSSION}

From these experiments it must be concluded that increase in isotonic contraction occurring after a premature beat and a compensatory pause cannot be explained by the Frank-Starling mechanism (Fig. 2). Siebens et al. (I I) and Lendrum and co-workers (I2) recently demonstrated, as well, that Starling's law of the heart cannot be applied to explain the increased force of the postextrasystolic contraction in the isometrically contracting canine ventricle. Siebens et al. (I I) also demonstrated a linear relationship between prematurity of the extra impulse and the force of contraction of the first postextrasystolic beat following a compensatory pause (Fig. 3).

A simple phenomenology for the occurrence of one enlarged contraction, following a premature beat plus a compensatory pause, may be derived from Table I and Fig. 4. If the increase in cycle length following a premature beat compensates the shortened cycle length

\section{REFERENCES}

I. Hoffman, B. F., E. Bindler, and E. E. Suckling. Am. J. Physiol. I 85: 95, I 956.

2. Rosin, H., and A. Farah. Am. J. Physiol. i 80: 75, i 955.

3. Kruta, V., And P. Braveny. Nature r $87: 327$, ig6o.

4. Bowditch, H. P. Arb. Physiol., Leipzig 6: I 39, I87 I.

5. Langendorff, O. Arch. Anat. u. Physiol. Abstr. 8: 284, I 885.

6. Langendorff, O. Arch. ges. Physiol., Pfüger's 70: 473, I898.

7. Frank, O. Z. Biol. 32: 370, I 895 (translated in a special article by C. B. Chapman and E. Wasserman: Am. Heart J. 58: 282, I 959 ).

8. Starling, E. H. The Lineacre Lecture on the Law of the Heart (1915). London: Longmans, I9I8.

9. Wiggers, C. J. Am. J. Physiol. 73: 346, I925.

ro. Wiggers, C. J. Circulatory Dynamics (Physiologic Studies). New York: Grune and Stratton, I $95^{2 .}$ preceding it, the enlargement of the postextrasystolic contraction compensates the smaller extrasystolic beat. In general it can be stated that average contraction height is constant at a constant net rate, whether the rhythm is regular or not. This is in agreement with Braveny's observations ( 18 ).

It is demonstrated in Fig. 5 that potentiation following an interpolating premature beat is different from potentiation following a premature beat plus a compensatory pause. This point is a common cause of misunderstanding and confusion. Owing to the very short refractory period of isolated myocardial muscle, every premature beat necessarily interpolates if no precautions are made to prevent it. Therefore, postextrasystolic potentiation in isolated myocardial strips is often different from postextrasystolic potentiation in intact hearts. We demonstrated that an interpolating premature beat gives rise to potentiation not to be distinguished from poststimulatioe potentiation (Fig. 6). Hoffman and colleagues (I) have already observed that the potentiating effect of extrasystoles in isolated papillary muscle resembles poststimulation potentiation. As a matter of fact, an interpolating premature beat causes a temporary increase of rate.

i i. Siebens, A. A., B. F. Hoffman, P. F. Cranefield, and C. McC. Brooks. Am. J. Physiol. 197: 97 I, 1959.

12. Lendrum, B., H. Feinberg, E. Boyd, and L. N. Katz. Am. J. Physiol. ig9: II I5, ig6o.

13. Hajdu, S., and E. Leonard. Pharmacol. Revs. 2: i73, 1959.

I 4. Langendorff, O. Arch. ges. Physiol., Pflüger's 6i : 291 , i 895.

i 5. Meijler, F. L., A. F. Willebrands, and Durrer. Circulation Research 8: 44, Ig6o.

i6. Durrer, D., J. Büller, P. Graaff, G. I. Lo, and F. L. MEIjLer. Circulation Research 9: 29, I96 I.

I7. VAN DAM, R. T. Experimenteel onderzoek naar het prikkelbaarheidsverloop van de hartspier (Thesis). Amsterdam: Poortpers, I 960.

18. Braveny, P. Scripta Medica 32: I94, I 959. 